\title{
Neutron waveguides in neutron optics: Green's functions formalism with Dirichlet boundary conditions
}

\author{
I. Molina de la Peña $\mathbb{1}^{a}$, M. L. Calvo $\mathbb{( 1 0}^{a}$ and R. F. Alvarez-Estrada $\mathbb{1}^{b}$ \\ ${ }^{a}$ Facultad de Ciencias Físicas, Departamento de Óptica, Universidad Complutense, Madrid, Spain; ${ }^{\text {b}}$ Facultad de Ciencias Físicas, Departamento \\ de Física Teórica, Universidad Complutense, Madrid, Spain
}

\begin{abstract}
In neutron optics, we analyse the propagation of slow (thermal) neutrons along a semi-infinite waveguide limited by a large repulsive potential (reminding the propagation of light along an optical fibre). We set an ideal case of a straight empty two-dimensional semi-infinite waveguide limited by an infinitely repulsive potential (Dirichlet conditions on its boundaries). The neutron wave function is given through an integral representation involving the incoming wave with energy $E$, Green's function and certain functions defined on the boundaries. The latter functions follow from the Dirichlet conditions, thereby proposing a new formalism in neutron optics. We develop various approximations (through Fourier and Hilbert transforms) and numerical computations. We get: (a) the expected extinction of the incoming wave for very large penetration into the waveguide, (b) the generation of propagation modes and their number for suitably large penetration, as $E$ increases, (c) an estimate of the critical angle.
\end{abstract}

\section{ARTICLE HISTORY}

Received 12 April 2020

Accepted 16 June 2020

\section{KEYWORDS}

Green-functions technique; integral transforms; neutron optics; thermal neutrons; waveguides; thin films

\section{Introduction}

Neutron optics is the branch of physics dealing with the theory and applications of the wave behaviour of slow neutrons, the electrically neutral subatomic particles that are present in all atomic nuclei except those of ordinary hydrogen. Neutron optics involves studying the interactions of matter with a beam of free slow neutrons, much as spectroscopy represents the interaction of electromagnetic radiation with matter. A general reference on slow neutrons ( $n$ ) and their properties is [1]. The possibility of guided slow neutron waves propagating along thin films was proposed and discussed earlier in 1973 [2]. Waveguiding phenomenon with slow neutron optics is an interesting topic being considered for the purpose of possible applications in materials science and biomedicine [1-6]. In this paper, we introduce a formalism based upon Green's functions and Dirichlet boundary conditions to formulate the propagation modes in neutron waveguides with small transverse cross sections. This formalism is an approach with the aim to implement specific numerical computation and to interpret the modal structure. The paper is organized as follows: it starts with the introduction, then, the formulation of the problem proposes the type of solution from stationary Schrödinger equation. The next subsection is devoted to the physical system description introducing the general integral equation based upon Green's functions and introducing, as well, certain functions defined on the boundaries and required to arrive at a rather feasible description of the modes propagation. The section dedicated to the methods contains seven subsections, starting from the Dirichlet boundary conditions, some approximations and arriving at a convenient formulation based upon Fourier and Hilbert transforms. The next section is devoted to the extinction phenomenon of the incoming wave for deep penetration into the waveguide that has to be demonstrated for the sake of consistency. Then, the section containing the results displays the possible interpretations and description following from the previous formalism: propagation modes, numerical computations aimed to justify the consistency of those approximations and approximately correct predictions of the number of propagation modes and of the critical angle associated to the semi-infinite waveguide. The paper ends with the discussion (including a physical justification, a posteriori, of our approach through infinitely repulsive potentials and Dirichlet boundary conditions), having in mind possible future work. The work is completed with an appendix (dealing with the conservation of the probability flux and providing a

CONTACT I. Molina de la Peña imolinap@ucm.es Facultad de Ciencias Físicas, Departamento de Óptica, Universidad Complutense, Madrid 28040, Spain 
consistent basis for approximations carried out in the main text).

\section{Formulation of the problem}

The wave function $\Psi(\boldsymbol{x}, t)$ for a slow (thermal) neutron at the position $\boldsymbol{x}$ (used here, generically, to denote one, two or three spatial dimensions) at time $t$, as the former propagates through the medium, satisfies the Schrödinger equation:

$$
\begin{gathered}
{\left[\frac{-\hbar^{2}}{2 m} \nabla^{2}+V(\boldsymbol{x}, t)\right] \Psi(\boldsymbol{x}, t)=i \hbar \frac{\partial \Psi(\boldsymbol{x}, t)}{\partial t}} \\
\begin{array}{l}
\text { Please, Bold } \\
\text { the "x" }
\end{array}
\end{gathered}
$$

$\hbar$ is Planck's consta the "X" n mass, $\nabla^{2}$ is the Laplacian and $V(x, t)$ is the effective optical potential due to the nuclei in the medium on the slow neutrons.

As the potential $V(\boldsymbol{x}, t)$ on the neutron during its displacement through media is usually $t$-independent, by factorizing the $t$-dependence, Equation (1) becomes an eigenvalue problem for the stationary wave equation:

$$
\begin{gathered}
{\left[\frac{-\hbar^{2}}{2 m} \nabla^{2}+V(\boldsymbol{x})-E\right] \varphi(\boldsymbol{x})=0} \\
E=\frac{2 \pi^{2} \hbar^{2}}{m \lambda_{d b} b^{2}}
\end{gathered}
$$

where $\lambda_{d b}$ is its de Broglie wavelength. Equation (2) has analogies with scalar wave equations used for classical electromagnetic fields deduced from Maxwell's equations, and can be exploited for characterizing the behaviour of a neutron beam. In particular, those phenomena associated with total internal reflection in tubes and neutron diffraction may be well characterized by setting an approximately constant ('optical') potential [1-5] into Equation (2) for describing the interaction with the homogeneous media:

$$
V=\frac{2 \pi \hbar^{2}}{m} b \rho
$$

$b$ is the coherent amplitude for the scattering of a slow neutron by a nucleus in the material. $b$ depends only on the material and isotope. $\rho$ is the density of nuclei per unit volume, of order $10^{22}$ (nuclei/ $\mathrm{cm}^{3}$ ). In general, disjoint spatial regions have different values for the product $b \rho$.

We remind here theoretical proposals, for exciting propagation modes for slow neutrons, by Wames and Sinha for thin planar films in 1973 [2] and by Calvo and Álvarez-Estrada in 1984 and 1986 [6,7] for thin fibres. Additionally, in 1994, Feng et al. experimentally demonstrated confined propagation of modes for slow neutrons in thin titanium films [8]. For further significant works on confined thermal neutron propagation in thin planar waveguides, also in the framework of neutron optics, see the other references in [8-14]. Other important studies are those of Kumakhov et al. [15] and Chen et al. [16], both in 1992, establishing experimentally the focusing of slow neutrons by using polycapillary glass fibre.

For confined propagation, the beam wavelength is close to the characteristic length scales of the system through which the neutron wave propagates (say, transverse sizes of thin films or fibres).

We shall quote here the Green's functions approach previously developed by Balian and Bloch [17] in a different physical context, namely, the analysis of the density of states for a large atomic nucleus. In that paper, the authors obtained integral equations involving Green's functions both for Dirichlet and Neumann boundary conditions, and developed iterative algorithms for their computation.

We propose here a non-trivial extension of the mathematical formulation, in [17], in order to solve the stationary wave equation for slow neutrons propagating in thin planar waveguides surrounded by regions with very large repulsive potentials, approximated through infinitely repulsive ones (modelled through Dirichlet boundary conditions) and by applying Green's functions methods. Our approach differs from that of Balian and Bloch in that our domain is not constrained to a large bounded spatial region (the atomic nucleus) but it is a semi-infinite region in space (the waveguide), so that an inhomogeneous term (the incoming wave) is included (absent in Balian and Bloch [17]).

To our knowledge, the latter formalism has not been applied previously in the literature in the context of confined waves propagation.

\section{Physical system description}

A rather general scalar wave equation takes the form of an inhomogeneous Helmholtz equation:

$$
\left[\nabla^{2}+k^{2}\right] \varphi(\boldsymbol{x})=F(\boldsymbol{x}) \varphi(\boldsymbol{x})
$$

$k^{2}$ is the wavevector associated to the neutron propagation in the medium and $F(\boldsymbol{x})$ represents a source term. In the subsequent development, we shall assume, for convenience, that there is no source term inside the region in which the neutron propagates $(F(\boldsymbol{x})=0)$ thus, Equation (5) reduces to a homogenous Helmholtz Equation (2) with $V=0$. Equivalently, we assume that $V=0$ (ie: no media) and thus, $k^{2}=\left(2 m E / \hbar^{2}\right)$

Since the problem is described by a scalar wavefunction, Green's identity for two functions $U$ and $W$ with continuous derivative in a region $\Omega$ and surrounded by 


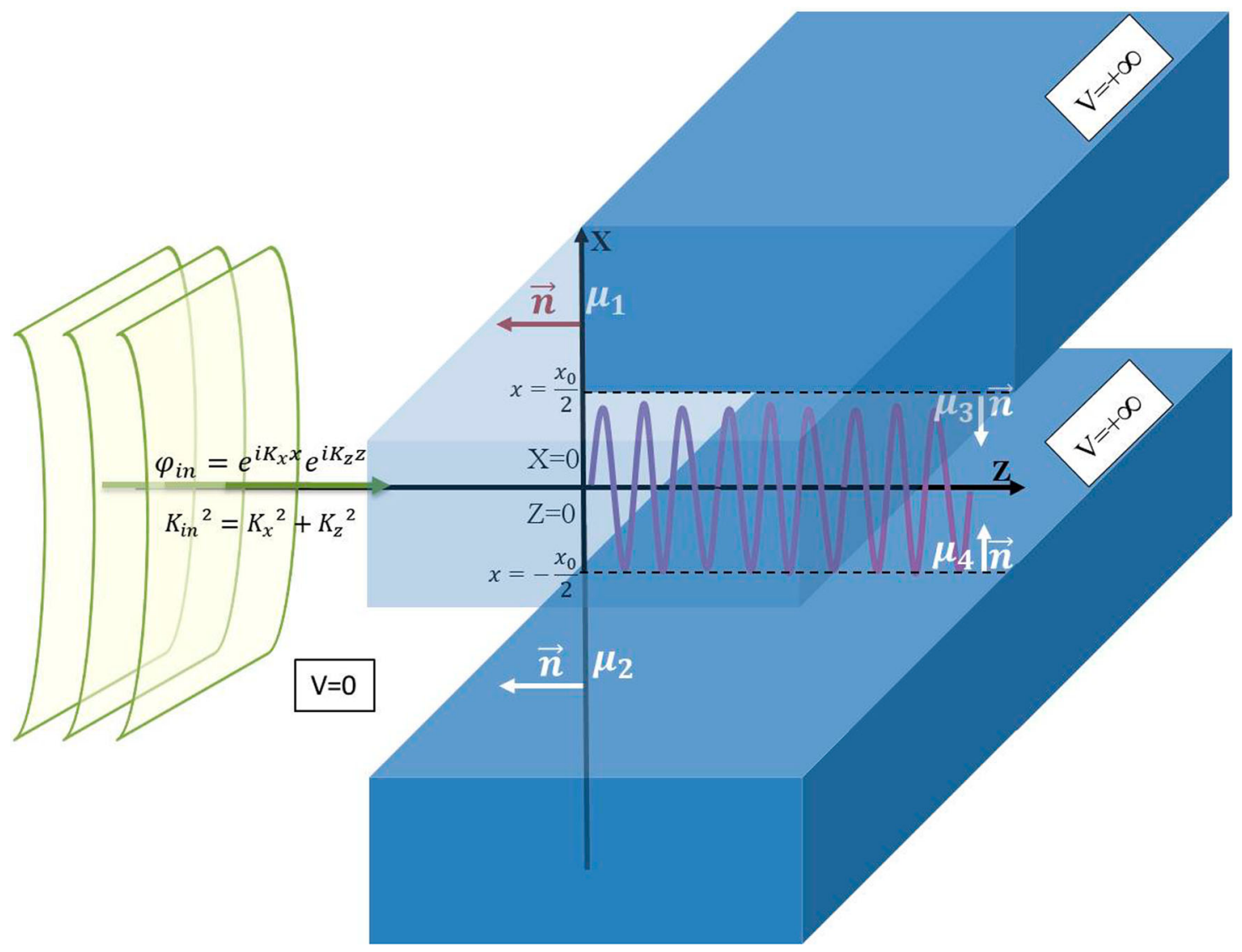

Figure 1. Schematic representation of the semi-infinite ideal waveguide with infinitely repulsive potential in the clad. Main features and parameters associated to the neutron confinement description are displayed. The analysis is carried out in two dimensions ( $x$ and $z$ ), for convenience.

the surface $\partial \Omega$ is: $\int_{\Omega}\left(U \nabla^{2} W-W \nabla^{2} U\right) d \Omega=$ $\int_{\partial \Omega}\left(U \frac{\partial W}{\partial \boldsymbol{n}}-W \frac{\partial U}{\partial \boldsymbol{n}}\right) d(\partial \Omega)$.

Applying to the previous expression the wave function that solves Equation (5), (i.e.: $U \equiv \varphi(\boldsymbol{x})$ ) and using the scalar Green's function $W \equiv G$ satisfying $\left[\nabla^{2}+k^{2}\right] G\left(\boldsymbol{x}, \boldsymbol{x}^{\prime}\right)=-\delta\left(\boldsymbol{x}-\boldsymbol{x}^{\prime}\right)$ we obtain:

$$
-\int_{\Omega} U\left(\boldsymbol{x}^{\prime}\right) \delta\left(\boldsymbol{x}-\boldsymbol{x}^{\prime}\right) d \Omega=\int_{\partial \Omega}\left(U \frac{\partial G}{\partial \boldsymbol{n}}-G \frac{\partial U}{\partial \boldsymbol{n}}\right) d(\partial \Omega)
$$

where $\delta\left(\boldsymbol{x}-\boldsymbol{x}^{\prime}\right)$ is the Dirac delta function. For a more detailed discussion see [18-20].

Let us set an ideal semi-infinite film waveguide as our region of interest, $\Omega$ (see Figure 1). For simplicity, we will focus on the $X Z$ plane by using a two-dimensional formulation. This region has an aperture $x_{0}$ along the $x$-axis and centred at the origin of coordinates. The waveguide extends along the $z$-axis, parallel to it, from $z=0$ to $z=+\infty$. The potential inside and along the guide is assumed to be $V=0(\rho b=0$, ie: no material $)$ as well as in the zone of incidence $z<0$. The clad of the waveguide, say the region $x \geq+x_{0} / 2, z \geq 0$ and $x \leq$ $-x_{0} / 2, z \geq 0$ is assumed to be an infinite ideal repulsive potential $V=+\infty$ and thus it is formulated by imposing Dirichlet boundary conditions as $(x, z)$ approaches the four walls of the guide; $\left(x \geq+x_{0} / 2, z=0\right),(x \leq$ $\left.-x_{0} / 2, z=0\right),\left(x=+x_{0} / 2, z \geq 0\right)$ and $\left(x=-x_{0} / 2, z \geq\right.$ $0)$. The four walls are included into the boundary $\partial \Omega$.

Figure 1 displays the considered geometrical model. We assume an incoming plane wave striking the aperture from $z=-\infty$ in the form of $\varphi_{i n}(x, z)=e^{i K_{x} x} e^{i K_{z} z}$. Notice that $K_{\text {in }}$ is bounded by $2 m E / \hbar^{2}$ for real modes.

We will find wavefunctions fulfilling Equation (5) for $V(\boldsymbol{x})=0$ for the region $z<0$ (the zone of incidence) and $-\left(x_{0} / 2\right) \leq x \leq\left(x_{0} / 2\right), z>0$ (the waveguide). For that purpose, we will make use of Equation (6) and [17]:

$$
\begin{aligned}
\varphi(x, z)= & \varphi_{\text {in }}(x, z)-\left.\int_{x_{0} / 2}^{\infty} d x^{\prime} \frac{\partial G\left(x-x^{\prime}, z-z^{\prime}\right)}{\partial z^{\prime}}\right|_{z^{\prime}=0} \mu_{1}\left(x^{\prime}\right) \\
& -\left.\int_{-\infty}^{-x_{0} / 2} d x^{\prime} \frac{\partial G\left(x-x^{\prime}, z-z^{\prime}\right)}{\partial z^{\prime}}\right|_{z^{\prime}=0} \mu_{2}\left(x^{\prime}\right)
\end{aligned}
$$




$$
\begin{aligned}
& -\left.\int_{0}^{\infty} d z^{\prime} \frac{\partial G\left(x-x^{\prime}, z-z^{\prime}\right)}{\partial x^{\prime}}\right|_{x^{\prime}=x_{0} / 2} \mu_{3}\left(z^{\prime}\right) \\
& +\left.\int_{0}^{\infty} d z^{\prime} \frac{\partial G\left(x-x^{\prime}, z-z^{\prime}\right)}{\partial x^{\prime}}\right|_{x^{\prime}=-x_{0} / 2} \mu_{4}\left(z^{\prime}\right)
\end{aligned}
$$

In Equation (7) all terms on the right-hand side fulfil separately the Schrödinger Equation (2) for any arbitrary functions $\mu_{i}, i=1 \ldots 4$ defined in the corresponding waveguide wall. Moreover, additional conditions must be imposed to obtain the corresponding functions. The same will be well characterized, in corresponding section, once Dirichlet boundary conditions be imposed in the clad.

Notice that the inhomogeneous term, $\varphi_{\text {in }}(x, z)$ arises from the need of closing the region $\Omega$ at $-\infty$ that is, $\varphi_{\text {in }}(x, z)$ represents the contribution of $\int_{\partial \Omega^{\prime}}\left(U \frac{\partial G}{\partial \boldsymbol{n}}-G \frac{\partial U}{\partial \boldsymbol{n}}\right)$ $d(\partial \Omega)$ if $\partial \Omega^{\prime}$ is the remainder of the boundary $\partial \Omega$. It is well known that this corresponds with the incident wave $[18,19]$. This term is absent in Balian and Bloch formulation [17] their problem being constrained to a finite region of space (i.e. the atomic nucleus).

In Equation (7), $G\left(x-x^{\prime}, z-z^{\prime}\right)$ corresponds to Green's function associated to the propagation in two dimensions represented as a superposition of plane waves. A discussion can be seen at $[18,19]$ :

$$
\begin{aligned}
& G\left(x-x^{\prime}, z-z^{\prime}\right) \\
& \quad=-\int \frac{d K_{x}^{\prime} d K_{z}^{\prime}}{(2 \pi)^{2}} \frac{\exp i\left[K_{x}^{\prime}\left(x-x^{\prime}\right)+K_{z}^{\prime}\left(z-z^{\prime}\right)\right]}{E+i \varepsilon-\frac{\hbar^{2}}{2 m}\left(K_{x}^{\prime 2}+K_{z}^{\prime 2}\right)}
\end{aligned}
$$

where $E=\left(\hbar^{2} K_{\text {in }}^{2} / 2 m\right)$ is the total energy of the incident wave. Notice that $\varepsilon>0$ is an infinitesimal term. It is understood that the limit $\varepsilon \rightarrow 0$ is taken as ending the calculations. We shall introduce the incoming wavevector as: $\vec{K}_{\text {in }}=\left(K_{x}, K_{z}\right)$.

\section{Methods}

\section{Dirichlet boundary conditions}

By imposing Dirichlet boundary conditions at the clad corresponding boundaries, the mathematical representation determining all functions $\mu_{i}$ are obtained. They represent the exact solution with further conditions for computational analysis. Thus, the wavefunction limits in the boundaries have to be calculated. For instance, for boundary condition yielding $\mu_{3}(z)$, one has to impose values at $x=x_{0} / 2$ :

$$
\varphi\left(\frac{x_{0}}{2}-\epsilon, z\right) \underset{\epsilon \rightarrow 0^{+}}{\rightarrow} 0, \forall z>0
$$

Then, by considering Equation (7), we particularize for $x=x_{0} / 2$. Notice that all terms are not singular at $x=$ $x_{0} / 2$ except for the one associated to $\mu_{3}\left(z^{\prime}\right)$. The limiting value of any non-singular terms turns out to coincide with its value at the same point in the wall. We will concentrate in the term associated to function $\mu_{3}(z)$ in the corresponding wall:

$$
\begin{aligned}
& \left.\frac{\partial G\left(\frac{x_{0}}{2}-x^{\prime}, z-z^{\prime}\right)}{\partial x^{\prime}}\right|_{x^{\prime}=\frac{x_{0}}{2}} \\
& =-\int_{-\infty}^{\infty} \frac{d K_{z}^{\prime}}{2 \pi} e^{i K_{z}^{\prime}\left(z-z^{\prime}\right)} \\
& \quad \times \int_{-\infty}^{\infty} \frac{d K_{x}^{\prime}}{2 \pi} \frac{\left(-i K_{x}^{\prime}\right) e^{i K_{x}^{\prime}\left(\frac{x_{0}}{2}-\frac{x_{0}}{2}\right)}}{E+i \varepsilon-\frac{\hbar^{2}}{2 m}\left(K_{x}^{\prime 2}+K_{z}^{\prime 2}\right)}=0
\end{aligned}
$$

following by symmetric integration over $K_{x}^{\prime}$. The result implies that the function $\mu_{i}$ associated to that wall does not contribute to the final solution. This is a natural conclusion of Green's theorem since we have already chosen the formulation corresponding to Dirichlet boundary conditions.

Moreover, if we calculate: $\lim _{\epsilon \rightarrow 0} \varphi\left(x=\frac{x_{0}}{2}-\epsilon, z\right)$ the result differs from the one following from Equation (10). By concentrating in the term associated to $\mu_{3}(z)$ and calculating this limit by applying residue integration:

$$
\begin{aligned}
\lim _{\epsilon \rightarrow 0^{+}} & \left.\frac{\partial G\left(\frac{x_{0}}{2}-\epsilon-x^{\prime}, z-z^{\prime}\right)}{\partial x^{\prime}}\right|_{x^{\prime}=\frac{x_{0}}{2}} \\
= & \lim _{\epsilon \rightarrow 0^{+}}-\int_{-\infty}^{\infty} \frac{d K_{z}^{\prime}}{2 \pi} e^{i K_{z}^{\prime}\left(z-z^{\prime}\right)} \\
& \times \int_{-\infty}^{\infty} \frac{d K_{x}^{\prime}}{2 \pi} \frac{\left(-i K_{x}^{\prime}\right) e^{i K_{x}^{\prime}(-\epsilon)}}{E+i \varepsilon-\frac{\hbar^{2}}{2 m}\left(K_{x}^{\prime 2}+K_{z}^{\prime 2}\right)} \\
= & -\frac{2 m}{\hbar^{2}} \frac{1}{2} \delta\left(z-z^{\prime}\right)
\end{aligned}
$$

And thus:

$$
\begin{gathered}
\lim _{\varepsilon \rightarrow 0^{+}}-\left.\int_{0}^{\infty} d z^{\prime} \mu_{3}\left(z^{\prime}\right) \frac{\partial G\left(\frac{x_{0}}{2}-\epsilon-x^{\prime}, z-z^{\prime}\right)}{\partial x^{\prime}}\right|_{x^{\prime}=\frac{x_{0}}{2}} \\
=\lim _{\varepsilon \rightarrow 0^{+}}+\frac{2 m}{\hbar^{2}} \int_{0}^{\infty} d z^{\prime} \delta\left(z-z^{\prime}\right) \mu_{3}\left(z^{\prime}\right)=\frac{2 m}{\hbar^{2}} \frac{1}{2} \mu_{3}(z)
\end{gathered}
$$

Other terms are not singular at $x=x_{0} / 2$, for both cases with and without imposing the limit $\epsilon \rightarrow 0^{+}$, respectively. By collecting both results in Equation (10) and 
(12), the equation yielding $\mu_{3}(z)$ is obtained after imposing the Dirichlet condition, in Equation (9):

$$
\lim _{\epsilon \rightarrow 0^{+}} \varphi\left(\frac{x_{0}}{2}-\epsilon, z\right)-\varphi\left(\frac{x_{0}}{2}, z\right)=\frac{2 m}{\hbar^{2}} \frac{1}{2} \mu_{3}(z)
$$

Thus, the first term in the left-hand side of Equation (13) must equal 0, due to Equation (9). Notice that by virtue of Equation (10) the term corresponding to $\mu_{3}$ in the second term in the left-hand side of Equation (13) equals 0 in: $x=\left(x_{0} / 2\right)$, Moreover, the term in the right-hand side of Equation (13) is the only one contributing upon performing the indicated substraction in the left-hand side of Equation (13).

The remaining three boundary equations are treated similarly. Thus, the system of inhomogeneous linear integral equations for $\mu_{1}, \mu_{2}, \mu_{3}, \mu_{4}, \mu_{i}=f\left(\mu_{j}, \mu_{k}, \mu_{l}\right) ; i \neq$ $j, k, l$, is obtained:

$$
\begin{aligned}
\frac{1}{2} \frac{2 m}{\hbar^{2}} & \mu_{1}(x) \\
= & -\varphi_{\text {in }}(x, 0)+\left.\int_{0}^{\infty} d z^{\prime} \mu_{3}\left(z^{\prime}\right) \frac{\partial G\left(x-x^{\prime},-z^{\prime}\right)}{\partial x^{\prime}}\right|_{x^{\prime}=\frac{x_{0}}{2}} \\
& -\left.\int_{0}^{\infty} d z^{\prime} \mu_{4}\left(z^{\prime}\right) \frac{\partial G\left(x-x^{\prime},-z^{\prime}\right)}{\partial x^{\prime}}\right|_{x^{\prime}=-\frac{x_{0}}{2}}, \text { for } x \geq x=\frac{x_{0}}{2}
\end{aligned}
$$

$$
\begin{aligned}
& \frac{1}{2} \frac{2 m}{\hbar^{2}} \mu_{2}(x) \\
& =-\varphi_{\text {in }}(x, 0)+\left.\int_{0}^{\infty} d z^{\prime} \mu_{3}\left(z^{\prime}\right) \frac{\partial G\left(x-x^{\prime},-z^{\prime}\right)}{\partial x^{\prime}}\right|_{x^{\prime}=\frac{x_{0}}{2}} \\
& \quad-\left.\int_{0}^{\infty} d z^{\prime} \mu_{4}\left(z^{\prime}\right) \frac{\partial G\left(x-x^{\prime},-z^{\prime}\right)}{\partial x^{\prime}}\right|_{x^{\prime}=-\frac{x_{0}}{2}} \text { for } x \leq-\frac{x_{0}}{2} \\
& \frac{1}{2} \frac{2 m}{\hbar^{2}} \mu_{3}(z) \\
& =-\varphi_{\text {in }}\left(\frac{x_{0}}{2}, z\right)+\left.\int_{\frac{x_{0}}{2}}^{\infty} d x^{\prime} \mu_{1}\left(x^{\prime}\right) \frac{\partial G\left(\frac{x_{0}}{2}-x^{\prime}, z-z^{\prime}\right)}{\partial z^{\prime}}\right|_{z^{\prime}=0} \\
& \quad+\left.\int_{-\infty}^{-\frac{x_{0}}{2}} d x^{\prime} \mu_{2}\left(x^{\prime}\right) \frac{\partial G\left(\frac{x_{0}}{2}-x^{\prime}, z-z^{\prime}\right)}{\partial z^{\prime}}\right|_{z^{\prime}=0} \\
& \quad-\left.\int_{0}^{\infty} d z^{\prime} \mu_{4}\left(z^{\prime}\right) \frac{\partial G\left(\frac{x_{0}}{2}-x^{\prime}, z-z^{\prime}\right)}{\partial x^{\prime}}\right|_{x^{\prime}=-\frac{x_{0}}{2}}
\end{aligned}
$$

$$
\begin{aligned}
& \frac{1}{2} \frac{2 m}{\hbar^{2}} \mu_{4}(z) \\
& \quad=-\varphi_{\text {in }}\left(-\frac{x_{0}}{2}, z\right)+\left.\int_{\frac{x_{0}}{2}}^{\infty} d x^{\prime} \mu_{1}\left(x^{\prime}\right) \frac{\partial G\left(-\frac{x_{0}}{2}-x^{\prime}, z-z^{\prime}\right)}{\partial z^{\prime}}\right|_{z^{\prime}=0}
\end{aligned}
$$

$$
\begin{aligned}
& +\left.\int_{-\infty}^{-\frac{x_{0}}{2}} d x^{\prime} \mu_{2}\left(x^{\prime}\right) \frac{\partial G\left(-\frac{x_{0}}{2}-x^{\prime}, z-z^{\prime}\right)}{\partial z^{\prime}}\right|_{z^{\prime}=0} \\
& +\left.\int_{0}^{\infty} d z^{\prime} \mu_{3}\left(z^{\prime}\right) \frac{\partial G\left(-\frac{x_{0}}{2}-x^{\prime}, z-z^{\prime}\right)}{\partial x^{\prime}}\right|_{x^{\prime}=\frac{x_{0}}{2}}
\end{aligned}
$$

Equations (14)-(17) determine $\mu_{i}$ functions in terms of the incoming wave $\varphi_{\text {in }}(x, z)$ (the inhomogeneous term). Some useful approximations to the exact solution (series expansion, functional analysis, numerical methods, iterative methods, etc.) are applicable. Notice, in Balian and Bloch [17] use of analogous equations with other inhomogeneous terms (not representing any sort of incoming plane wave) led to the development of an iterative algorithm simulating certain properties of an atomic nucleus.

\section{Analytical study of the system}

To our current study, obtaining an exact solution for the proposed system of Equations (14)-(17) leading to the characterization of the field, $\varphi(x, z)$ is not feasible. In general, no analytical representation for the functions $\mu_{i}(i=1 \ldots 4)$ is able to be computed. Moreover, a rigorous control of the convergence of the successive iterations of the system, Equations (14)-(18), or application of Fredholm's theory is not easy to achieve. In this section, we propose simple analytical forms for the functions $\mu_{i},(i=1 \ldots 4)$ and related approximations in order to treat the proposed system. This is aimed to demonstrate the existence, for suitably large $E$, of trapped energy levels inside the waveguide (propagation modes). The computation of the energy levels achieved in the guide rely on finding some convenient relationships between the characterization of the system and some mathematical transforms related to optics, in particular Fourier and Hilbert transforms, as well.

\section{Alternative representation for the wavefunction}

Let us introduce $\Lambda_{i},(i=1 \ldots 4)$ for each of the four integrals related to $\mu_{i},(i=1 \ldots 4)$ in order to deal with them separately. By applying the residue theorem as in Equation (11), Equation (7) reads (with no approximations):

$$
\varphi(x, z)=\varphi_{\text {in }}(x, z)+\Lambda_{1}+\Lambda_{2}+\Lambda_{3}+\Lambda_{4}
$$

On the one hand, with the choice $K_{z}^{\prime}=+\sqrt{\frac{2 m E}{\hbar^{2}}-K_{x}^{\prime 2}}$ for $z<$ or $>0$ : 


$$
\begin{aligned}
& \Lambda_{1}= \pm \frac{2 m}{\hbar^{2}} \frac{1}{2} \int \frac{d K_{x}^{\prime}}{2 \pi} \int_{x_{0} / 2}^{\infty} d x^{\prime} \mu_{1}\left(x^{\prime}\right) e^{i K_{x}^{\prime}\left(x-x^{\prime}\right)} \mathrm{e}^{\mp i z K_{z}^{\prime}} \\
& \Lambda_{2}= \pm \frac{2 m}{\hbar^{2}} \frac{1}{2} \int \frac{d K_{x}^{\prime}}{2 \pi} \int_{-\infty}^{-x_{0} / 2} d x^{\prime} \mu_{2}\left(x^{\prime}\right) e^{i K_{x}^{\prime}\left(x-x^{\prime}\right)} \mathrm{e}^{\mp i z K_{z}^{\prime}}
\end{aligned}
$$

On the other hand, for $z>0$ and $K_{x}^{\prime}=+\sqrt{\frac{2 m E}{\hbar^{2}}-K_{z}^{\prime 2}}$

$$
\begin{aligned}
& \Lambda_{3}=\frac{2 m}{\hbar^{2}} \frac{1}{2} \int \frac{d K_{z}^{\prime}}{2 \pi} \int_{0}^{\infty} d z^{\prime} \mu_{3}\left(z^{\prime}\right) \mathrm{e}^{i K_{z}^{\prime}\left(z-z^{\prime}\right)} e^{-i K_{x}^{\prime}\left(x-x_{0} / 2\right)} \\
& \Lambda_{4}=\frac{2 m}{\hbar^{2}} \frac{1}{2} \int \frac{d K_{z}^{\prime}}{2 \pi} \int_{0}^{\infty} d z^{\prime} \mu_{4}\left(z^{\prime}\right) \mathrm{e}^{i K_{z}^{\prime}\left(z-z^{\prime}\right)} e^{i K_{x}^{\prime}\left(x+x_{0} / 2\right)}
\end{aligned}
$$

We omit the corresponding expressions for $\Lambda_{3}$ and $\Lambda_{4}$ for $z<0$ since we shall not considered them. Integrations over wavevector are carried out in $(-\infty,+\infty)$.

Notice that since $K_{i n}^{2}=\frac{2 m E}{\hbar^{2}}=K_{x}^{\prime 2}+K_{z}^{\prime 2}$ there is a constraint in the magnitude of certain exponentials when we perform the integration over the corresponding wavevector $\left(K_{x}^{\prime}\right.$ or $\left.K_{z}^{\prime}\right)$. Consequently, those values such that that $K_{x}^{\prime}>\frac{2 m E}{\hbar^{2}}$ and so for $K_{z}^{\prime}$ imply evanescent waves (i.e.: modes in the waveguide with energy higher than the incoming particle). See Figure 2:

It is physically acceptable to suppose that the main expected phenomena are: reflection of a part of the incoming wave for $z<0$, extinction of the incoming wave and penetration of propagation modes of the wave along the aperture for $z>0$, for adequately large $E$. Thus, generation of certain diffraction of the incoming wave by the finite aperture (the waveguide entrance) arises. This will be taken into account by means of the appropriate choice of values $\mu_{1}\left(x^{\prime}\right)$ and $\mu_{2}\left(x^{\prime}\right)$, and the waveguiding effects (confinement) due to the presence of the clad, related to $\mu_{3}\left(z^{\prime}\right)$ and $\mu_{4}\left(z^{\prime}\right)$, for adequately large $E$.

\section{An approximate analytical representation for the auxiliary functions $\mu_{i}$}

At this point, we make a physical ansatz for the functions $\mu_{i},(i=1 \ldots 4)$ for generic incoming neutron energy $E$ and check whether the final result obeys, approximately, the boundary conditions of the problem.

One can argue that, in a first approximation, the contributions of the terms associated to $\mu_{3}\left(z^{\prime}\right)$ and $\mu_{4}\left(z^{\prime}\right)$ in Equations (14) and (15), respectively, can be neglected and set for the value of the auxiliary function for $x^{\prime}$ positive and large and $-x^{\prime}$ positive and large, respectively in their corresponding ranges:

$$
\frac{1}{2} \frac{2 m}{\hbar^{2}} \mu_{1}\left(x^{\prime}\right) \simeq \frac{1}{2} \frac{2 m}{\hbar^{2}} \mu_{2}\left(x^{\prime}\right) \simeq-\varphi_{\text {in }}\left(0, x^{\prime}\right)=-e^{i K_{x} x^{\prime}}
$$

Notice that this condition characterizes the system for any arbitrary angle of incidence of the incoming wave.

The above approximation in Equation (23) is consistent with the conservation of the probability flux along the $z$ axis, as outlined in Appendix. It is also consistent with total reflection in $z<0$, for $x>0$ and large (for $\mu_{1}$ ) and $x<0$ and large (for $\mu_{2}$ ).

On searching for approximations for $\mu_{3}\left(z^{\prime}\right)$ and $\mu_{4}\left(z^{\prime}\right)$, we shall turn to Equations (16) and (17). It can be seen that, considering large $z>0$, with $z \gg x_{0}$, the contributions associated with $\mu_{1}(x)$ and $\mu_{2}(x)$ cannot be neglected in the latter equations. In fact, once those terms are computed with the approximations used in Equation (23) and as shown in Equation (25) (next section) they will not lead to cancellation of the incoming wave. In search of feasible approximate physical solutions, we shall also introduce the functions $\mu_{3}\left(z^{\prime}\right)$ and $\mu_{4}\left(z^{\prime}\right)$ as:

$$
\frac{2 m}{\hbar^{2}} \mu_{3,4}\left(z^{\prime}\right)=-e^{i K_{z} z^{\prime}}+\mu_{+,-} e^{i q z^{\prime}}
$$

$\mu_{+}$and $\mu_{-}$are suitable normalization constants to be evaluated later and $q$ is an arbitrary spatial frequency. In Equation (24) the first term will contribute (at least partially) to cancel out the incident wave (extinction) and the second one will represent the propagation modes in the guide, once its wavevector $q$ be determined by imposing Dirichlet boundary conditions. In a more general ansatz, in the right-hand side of Equation (24) one should write a sum of terms under the form $\mu_{+,-} e^{i q z^{\prime}}$ (one of each $q$ with different $\mu_{+,-}$depending on $q$ ). Having the latter in mind, and for the sake of simplicity, we shall continue with Equation (24).

\section{Study of the penetration along the waveguide $\left(\Lambda_{1}+\Lambda_{2}\right.$ for $\left.z>0\right)$}

The second and third terms in Equation (18), $\Lambda_{1}$ and $\Lambda_{2}$, represent the interaction of the incoming wave with the entrance in the waveguide.

Recalling Equations (19), (20) and (23) for $z>0$ :

$$
\begin{aligned}
\Lambda_{1}+\Lambda_{2}= & -\frac{2 m}{\hbar^{2}} \frac{1}{2} \int \frac{d K_{x}^{\prime}}{2 \pi}\left[\int_{x_{0} / 2}^{\infty} d x^{\prime} \mu_{1}\left(x^{\prime}\right) e^{i K_{x}^{\prime} x^{\prime}}\right. \\
& \left.+\int_{-\infty}^{-x_{0} / 2} d x^{\prime} \mu_{2}\left(x^{\prime}\right) e^{i K_{x}^{\prime} x^{\prime}}\right] e^{i x K_{x}^{\prime}} \mathrm{e}^{i z K_{z}^{\prime}}
\end{aligned}
$$






Figure 2. Representation of the phase space integration region for Equations (19)-(22). Two zones defining propagating and evanescent waves depending on impinging energy are displayed.

$$
\begin{aligned}
& \approx\left[\int_{-\infty}^{\infty} d x^{\prime} e^{i\left(K_{x}-K_{x}^{\prime}\right) x^{\prime}}\right] \int \frac{d K_{x}^{\prime}}{2 \pi} e^{i K_{x}^{\prime} x} \mathrm{e}^{i z K_{z}^{\prime}} \\
& =e^{i K_{x} x} e^{i z \sqrt{\frac{2 m E}{\hbar^{2}}-K_{x}^{2}}}=\varphi_{\text {in }}(x, z)
\end{aligned}
$$

In Equation (25), we are dropping inessential terms associated to the diffraction phenomenon taking place as the neutron beam is confined, and thus, considering only modal contributions. Notice, the diffraction will produce additional noise in the waveguide diminishing as neutrons propagate along the same. Therefore, this approximation is valid for long enough $z$ values. We shall analyse some physical effects neglected in the approximation displayed in Equation (25).

\section{Analysis of the approximation in Equation (25)}

In this subsection, we shall interpret the physical meaning of the terms neglected in Equation (25). Let us consider the full integrand in the integral over $K_{x}^{\prime}$ in the latter:

$$
\begin{gathered}
\int_{x_{0} / 2}^{\infty} d x^{\prime} \mu_{1}\left(x^{\prime}\right) e^{-i K_{x}^{\prime} x^{\prime}}+\int_{-\infty}^{-x_{0} / 2} d x^{\prime} \mu_{2}\left(x^{\prime}\right) e^{-i K_{x}^{\prime} x^{\prime}} \\
=\left[\int_{-\infty}^{\infty} d x^{\prime} e^{-i K_{x}^{\prime} x^{\prime}}-\int_{-x_{0} / 2}^{x_{0} / 2} d x^{\prime} e^{-i K_{x}^{\prime} x^{\prime}}\right]
\end{gathered}
$$

The first term in the right-hand side of Equation (26) is the one we used in previous subsection. The second term represents the error made in that approximation and is the one we will analyse here. Indeed, the contribution of this term approaches to 0 for $z>0$ large enough.

Since the added term can be analytically integrated by introducing it as trivial connection in Equation (25), we have to demonstrate the following contribution tending to 0 for large $z>0$ :

$$
\begin{aligned}
& \int_{-\infty}^{\infty} \frac{d K_{x}^{\prime}}{K_{x}^{\prime}} e^{i K_{x}^{\prime} x}\left\{e^{-i K_{x}^{\prime} x_{0} / 2}-e^{i K_{x}^{\prime} x_{0} / 2}\right\} e^{i z \sqrt{\frac{2 m E}{\hbar^{2}}-K_{x}^{\prime 2}}} \rightarrow 0 \\
& \quad z \rightarrow+\infty
\end{aligned}
$$

For the sake of simplicity, we omit all constant factors, as we are interested in the function behaviour. In order to demonstrate the convergence of this expression (i.e. demonstrate that the approximation is suitable for $z$ sufficiently long) we consider only the first term in Equation (27), the reproduction of similar operations for the second term being trivial:

$$
\begin{aligned}
& \int_{-\infty}^{\infty} \frac{d K_{x}^{\prime}}{K_{x}^{\prime}} e^{i K_{x}^{\prime}\left(x-x_{0} / 2\right)} e^{i z K_{z}^{\prime}} \\
& =\int_{-\infty}^{\infty} \frac{d K_{x}^{\prime}}{K_{x}^{\prime}}\left[\cos \left(K_{x}^{\prime}\left(x-x_{0} / 2\right)\right)\right. \\
& \left.\quad+i \sin \left(K_{x}^{\prime}\left(x-x_{0} / 2\right)\right)\right] e^{i z \sqrt{\frac{2 m E}{\hbar^{2}}-K_{x}^{\prime 2}}}
\end{aligned}
$$

By invoking symmetric integration in the integrand, only the sine term will contribute with a non-vanishing result. Notice that the square root function is purely real 


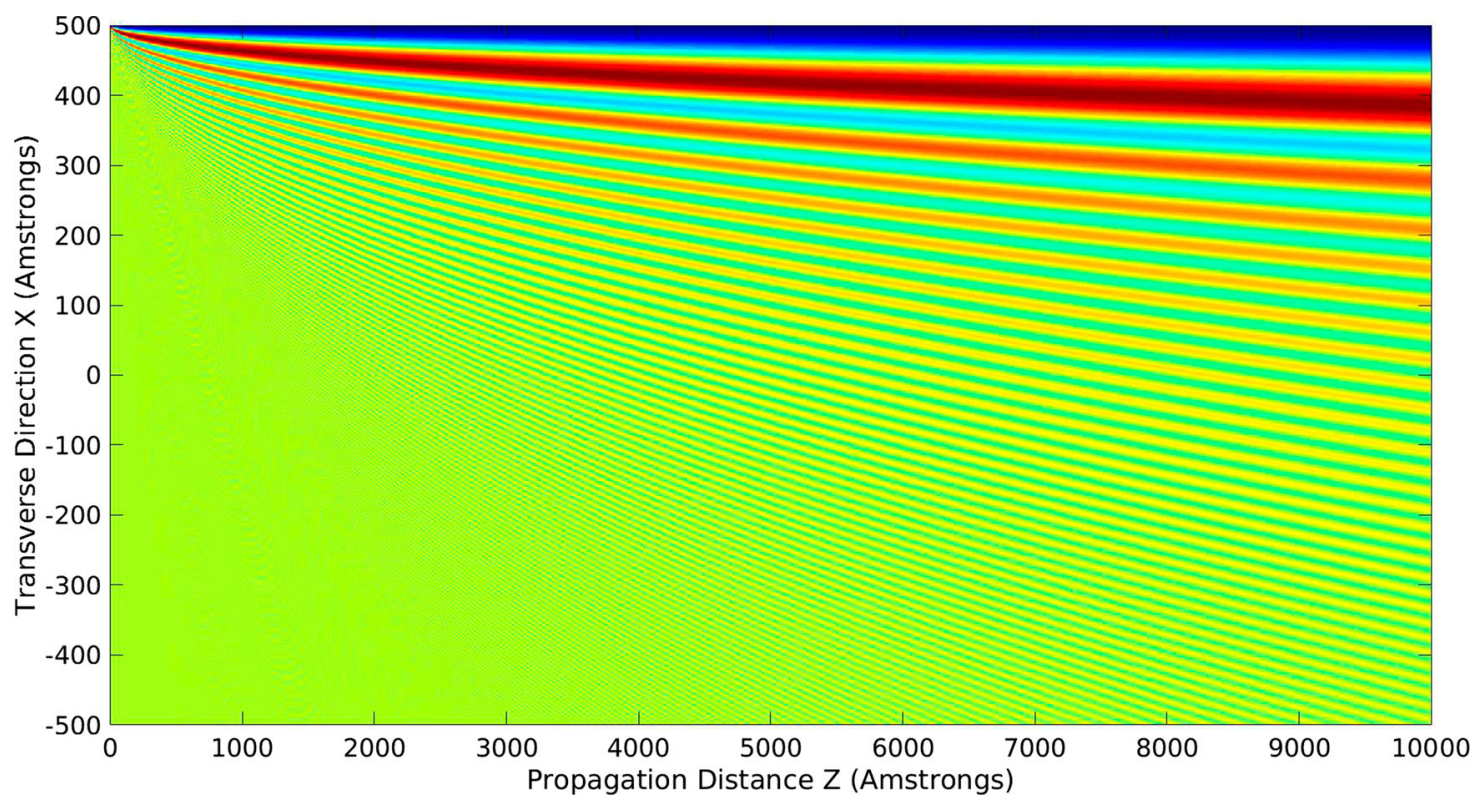

Figure 3. Representation of the absolute square value of Equation (30), simulated for an incident wave of $E=0.025 \mathrm{eV}$ and a guide aperture of $1000 \AA$

in the interval: $K_{x}^{\prime 2} \leq \frac{2 m E}{\hbar^{2}}=K_{i n}^{2}$. Then Equation (28) yields:

$$
\begin{aligned}
& \int_{-\infty}^{\infty} i \frac{d K_{x}^{\prime}}{K_{x}^{\prime}} \sin \left(K_{x}^{\prime}\left(x-x_{0} / 2\right)\right) e^{i z \sqrt{K_{i n}^{2}-K_{x}^{\prime 2}}} \\
& =\int_{-\infty}^{-K_{i n}} i \frac{d K_{x}^{\prime}}{K_{x}^{\prime}} \sin \left(K_{x}^{\prime}\left(x-x_{0} / 2\right)\right) e^{-z \sqrt{\left|K_{i n}^{2}-K_{x}^{\prime 2}\right|}} \\
& +\int_{-K_{i n}}^{K_{\text {in }}} i \frac{d K_{x}^{\prime}}{K_{x}^{\prime}} \sin \left(K_{x}^{\prime}\left(x-x_{0} / 2\right)\right) e^{i z \sqrt{K_{\text {in }}^{2}-K_{x}^{\prime 2}}} \\
& +\int_{K_{\text {in }}}^{\infty} i \frac{d K_{x}^{\prime}}{K_{x}^{\prime}} \sin \left(K_{x}^{\prime}\left(x-x_{0} / 2\right)\right) e^{-z \sqrt{\left|K_{\text {in }}^{2}-K_{x}^{\prime 2}\right|}}
\end{aligned}
$$

In Equation (29), the first and the third terms, respectively, vanish for large $z$. They represent the evanescent wave arising from the entrance of the guide.

The second term in Equation (29) can be viewed as a Fourier transform of the integrand. Just by performing the change of variable $\sqrt{K_{i n}-K_{x}^{\prime 2}}=-\tau$ and imposing infinite integration limits with the use of the rect function, then, this second term yields:

$$
\int_{-K_{\text {in }}}^{K_{i n}} i \frac{d K_{x}^{\prime}}{K_{x}^{\prime}} \sin \left(K_{x}^{\prime}\left(x-x_{0} / 2\right)\right) e^{i z \sqrt{K_{i n}^{2}-K_{x}^{\prime 2}}}
$$

$$
\begin{aligned}
= & F T\left\{\operatorname{rect}\left(\frac{\sqrt{K_{i n}^{2}-\tau^{2}}}{2 K_{i n}}\right) \cdot \frac{-\tau}{\sqrt{K_{i n}^{2}-\tau^{2}}}\left(x-x_{0} / 2\right)\right. \\
& \left.\times \frac{\sin \left(\left(x-x_{0} / 2\right) \sqrt{K_{i n}^{2}-\tau^{2}}\right)}{\left(x-x_{0} / 2\right) \sqrt{K_{i n}^{2}-\tau^{2}}}\right\}
\end{aligned}
$$

where FT denotes the Fourier Transform of the inner function. By applying Riemann-Lebesgue lemma [21], it can be shown that it tends to 0 for high $z$. The rect function is the standard window function.

As a more complete physical explanation, Equation (30) represents the diffraction produced by the aperture of the waveguide directly related with the Fourier transform of the incoming signal. Some modes are allowed in the phase space associated to the transform and thus, give rise to propagating waves. Meanwhile, those modes exceeding the incoming wave wavevector values, $\vec{K}_{i n}$, give rise to evanescent modes. This is consistent with Figure 2. A numerical representation is shown on Figure 3 where diffraction effect is enhanced:

The aperture diffraction is similar to a displacement of the $x$ axis in Equation (30). In Figure 4, one appreciates the function behaviour for three different apertures. As wave propagates along $z$ axis, the result is an oscillatory function reaching a maximum and, then, it decays asymptotically. The wider the aperture the longer the propagation in $z$ axis to reach this maximum.

Moreover, in Figure 5, we have performed a simulation of the position of this maximum as a function of 


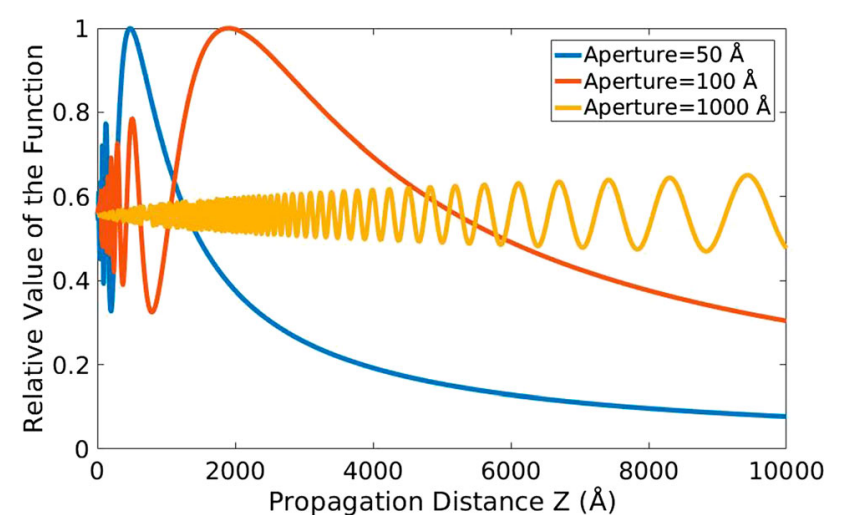

Figure 4. Representation of the absolute square value of Equation (30) centred in the $z$-axis, $\varphi(0, z)$ for an incident wave of $E=0.025 \mathrm{eV}$ in relation with different apertures.

the aperture. It is clear that the maximum, before which the function decays, is reached further in the waveguide as the aperture increases, which is the expected physical behaviour for the aperture diffraction.

\section{Study of wave confinement for $\mathbf{z}>0$ adequately larger than $x_{0}$}

The fourth and fifth terms in Equation (18), $\Lambda_{3}$ and $\Lambda_{4}$ respectively, will generate both the confinement in the wave as well as the extinction of the incoming wave.

From Equations (21) and (22) and having in mind Equation (24) one finds, for any real arbitrary spatial frequency, $q>0$ :

$$
\int \frac{d K_{z}^{\prime}}{2 \pi}\left[\int_{0}^{\infty} d z^{\prime} e^{i q z^{\prime}} e^{-i K_{z}^{\prime} z^{\prime}}\right] e^{i K_{z}^{\prime} z} e^{-i\left(x-x_{0} / 2\right) \sqrt{\frac{2 m E}{\hbar^{2}}-K_{z}^{\prime 2}}}
$$

The inner integral is indeed a Fourier transform, denoted by $F T$, of the product of a Heaviside step function, step $(z)$, and $e^{i q z^{\prime}}$ :

$$
\begin{aligned}
\int_{0}^{\infty} d z^{\prime} e^{i q z^{\prime}} e^{-i K_{z}^{\prime} z^{\prime}} & =F T\left[\operatorname{step}\left(z^{\prime}\right)\right]_{K_{z}^{\prime}-q} \\
& =\pi\left(\frac{1}{i \pi\left(K_{z}^{\prime}-q\right)}+\delta\left(K_{z}^{\prime}-q\right)\right)
\end{aligned}
$$

Notice that the Dirac delta function would be obtained in the case of performing the integral from $-\infty$ to $+\infty$ (i.e.: if the waveguide would be infinite). Consequently, the term $\frac{1}{i \pi\left(K_{z}^{\prime}-q\right)}$ arises from having a semi-infinite (say an initial aperture) waveguide at $z=0$. We will pay attention to $\frac{1}{i \pi\left(K_{z}^{\prime}-q\right)}$ term arising in Equation (32). By introducing it in Equation (31):

$$
\int \frac{d K_{z}^{\prime}}{2 \pi} \frac{e^{i K_{z}^{\prime} z}}{i\left(K_{z}^{\prime}-q\right)} e^{-i\left(x-x_{0} / 2\right) \sqrt{\frac{2 m E}{\hbar^{2}}-K_{z}^{\prime 1}}}
$$

We notice that Equation (33), is the Hilbert Transform of the product of two exponential functions, the Hilbert transform being defined by using the Cauchy principal value.

For simplicity, we denote $f\left(K_{z}^{\prime}\right)=e^{i K_{z}^{\prime} z}$ and $g\left(K_{z}^{\prime}\right)=$ $e^{-i\left(x-x_{0} / 2\right) \sqrt{\frac{2 m E}{\hbar^{2}}-K_{z}^{\prime 2}}}$. So, Equation (33) turns out to be:

$$
\frac{1}{i 2 \pi} \int d K_{z}^{\prime} \frac{f\left(K_{z}^{\prime}\right) g\left(K_{z}^{\prime}\right)}{\left(K_{z}^{\prime}-q\right)}=\frac{1}{2 i} \mathcal{H}\left[f\left(K_{z}^{\prime}\right) g\left(K_{z}^{\prime}\right)\right]
$$

here, $\mathcal{H}$ denotes the Hilbert transform operator. We remind here some useful properties of this transform. For more details, see the Bedrosian theorem [22] and [23,24].

Let $f\left(K_{z}^{\prime}\right)$ and $g\left(K_{z}^{\prime}\right)$ be complex functions in the $L^{2}(-\infty, \infty)$ of the real variable $K_{z}^{\prime}$, and let both $f\left(K_{z}^{\prime}\right)$ and $g\left(K_{z}^{\prime}\right)$ be analytic functions of $K_{z}^{\prime}$. Then:

$$
\begin{gathered}
\mathcal{H}\left[f\left(K_{z}^{\prime}\right) g\left(K_{z}^{\prime}\right)\right]=f(q) \mathcal{H}\left[g\left(K_{z}^{\prime}\right)\right]_{q}=g(q) \mathcal{H}\left[f\left(K_{z}^{\prime}\right)\right]_{q} \\
\mathcal{H}\left[\cos \left(K_{z}^{\prime} z\right)\right]=-\sin (q z) \\
\mathcal{H}\left[\sin \left(K_{z}^{\prime} z\right)\right]=\cos (q z)
\end{gathered}
$$

Then, combining Equations (31) to (37):

$$
\begin{aligned}
& \int \frac{d K_{z}^{\prime}}{2 \pi}\left[\int_{0}^{\infty} d z^{\prime} e^{i q z^{\prime}} e^{-i K_{z}^{\prime} z^{\prime}}\right] e^{i K_{z}^{\prime} z} e^{-i\left(x-x_{0} / 2\right) \sqrt{\frac{2 m E}{\hbar^{2}}-K_{z}^{\prime 2}}} \\
& =e^{i q z} e^{-i\left(x-x_{0} / 2\right) \sqrt{\frac{2 m E}{\hbar^{2}}-q^{2}}}
\end{aligned}
$$

\section{Extinction conditions: aproximate formulation}

By considering Equation (38), one will use the two respective contributions in Equation (24), namely, for the incoming $q=K_{z}>0$. and also for a generic $q>0$ (eventually associated to propagation modes). By combining Equation (18) with the corresponding results obtained in Equations (25), (32) and (38), one obtains the corresponding approximation following from the ansatz for the functions $\mu_{i}, i=1 \ldots 4$ (i.e.: $\frac{1}{2} \frac{2 m}{\hbar^{2}} \mu_{1}\left(x^{\prime}\right)=$ $\frac{1}{2} \frac{2 m}{\hbar^{2}} \mu_{2}\left(x^{\prime}\right)=-\varphi_{\text {in }}(x, 0)$ and $\frac{2 m}{\hbar^{2}} \mu_{3,4}\left(z^{\prime}\right)=-e^{i K_{z} z^{\prime}+}$ $\left.\mu_{+,-} e^{i q z^{\prime}}\right)$. The approximation for the whole Equation (18) reads, for suitably large penetration distance into the waveguide:

$$
\begin{aligned}
\varphi(x, z)= & \varphi_{i n}(x, z)+e^{i K_{x} x} e^{i K_{z} z} \\
& -e^{i K_{z} z}\left[e^{-i\left(x-\frac{x_{0}}{2}\right) K_{x}}+e^{i\left(x+\frac{x_{0}}{2}\right) K_{x}}\right] \\
& +e^{i q z}\left[\mu_{+} e^{-i\left(x-\frac{x_{0}}{2}\right) \sqrt{\frac{2 m E}{\hbar^{2}}-q^{2}}}\right. \\
& \left.+\mu_{-} e^{i\left(x+\frac{x_{0}}{2}\right) \sqrt{\frac{2 m E}{\hbar^{2}}-q^{2}}}\right]
\end{aligned}
$$




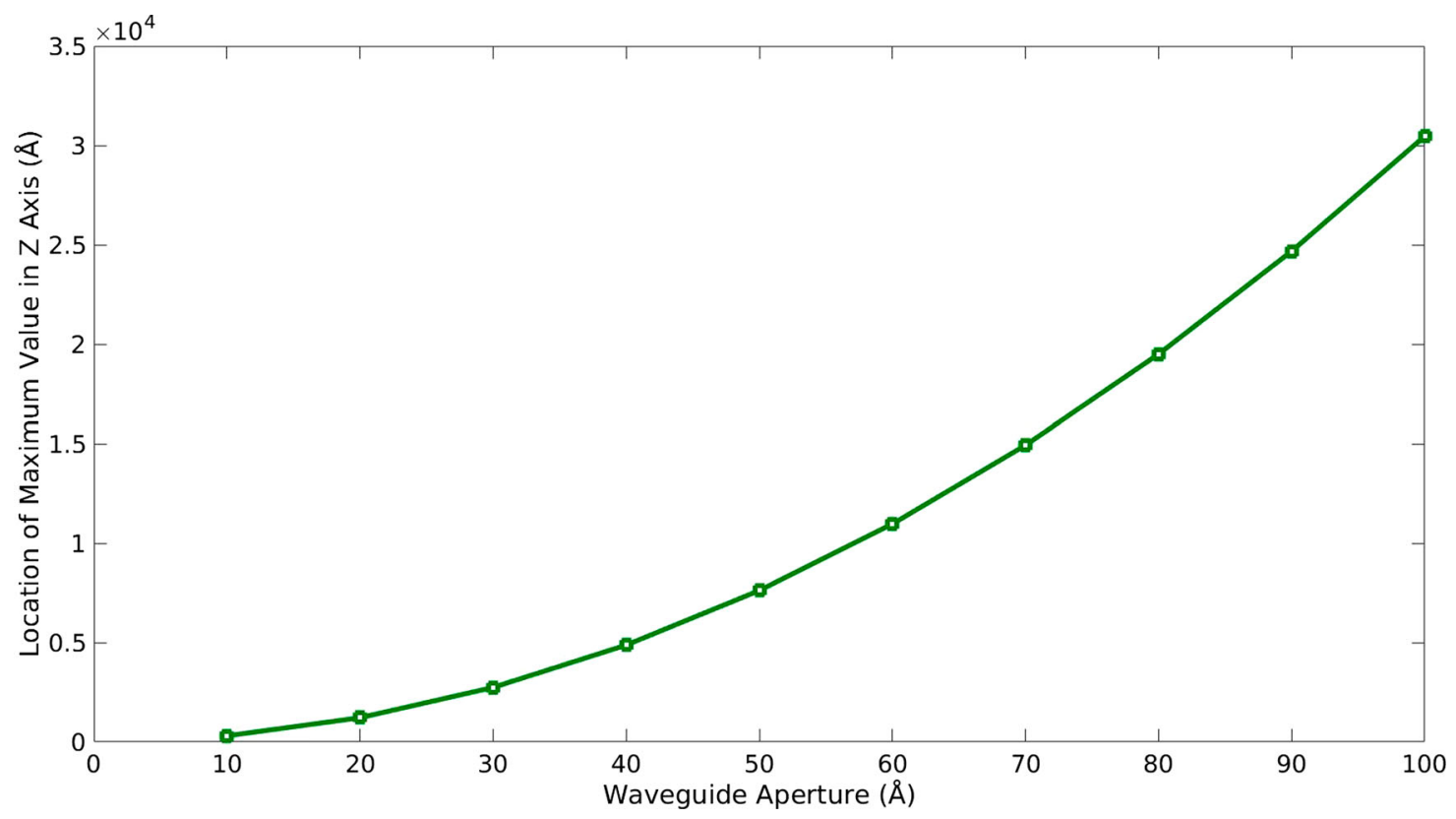

Figure 5. Representation of the $\mathrm{z}$ propagation distance versus waveguide aperture. Incident wave energy: $E=0.025 \mathrm{eV}$

If $K_{x}=0$ or if $K_{x} x_{0} \ll 1$, one has an approximate extinction of incoming wave and the approximate $\Lambda_{1}$ and $\Lambda_{2}$ with part of the contribution of $\Lambda_{3}$ and $\Lambda_{4}$. See also case for low energies in next section. Then:

$$
\begin{aligned}
\varphi(x, z) \simeq & e^{i q z}\left[\mu_{+} e^{-i\left(x-\frac{x_{0}}{2}\right) \sqrt{\frac{2 m E}{\hbar^{2}}-q^{2}}}\right. \\
& \left.+\mu_{-} e^{i\left(x+\frac{x_{0}}{2}\right) \sqrt{\frac{2 m E}{\hbar^{2}}-q^{2}}}\right]
\end{aligned}
$$

which is ready to discuss propagation modes for (suitably large) $z>0$, with a wavevector that must obey the constraint $\vec{q}=\left(q_{x}, q_{z}\right)=\left(\sqrt{\frac{2 m E}{\hbar^{2}}-q^{2}}, q\right)=$ $\left(\sqrt{K_{\text {in }}^{2}-q^{2}}, q\right)$. If $K_{x} x_{0} \ll 1$ does not hold, then, there is no such extinction in the framework of our approximations. Physically, as propagation modes are excited for adequately large $E$ they are, therefore, the only remaining contributions for deep penetration into the waveguide. The incoming wave has to cancel out there exactly with certain contributions from $\Lambda_{1}+\Lambda_{2}+\Lambda_{3}+\Lambda_{4}$ according to Equation (18). Such an exact extinction operates outside our approximations.

\section{Results}

\section{Analysis of allowable propagation modes and energy levels}

In order to obtain the propagation modes (energy levels) associated to the guide structure, one has to remind the fulfilment of Dirichlet conditions at $x= \pm x_{0} / 2$ for a particular wavevector $q$. It is trivial to notice that the imposition of these boundary conditions provides odd and even propagation modes in:

$$
\begin{gathered}
\varphi_{\text {odd }}(x, z)=-i \sin \left(\frac{2 n \pi x}{x_{0}}\right) e^{i z \sqrt{\frac{2 m E}{\hbar^{2}}-\left(\frac{2 \pi n}{x_{0}}\right)^{2}}} \\
\varphi_{\text {even }}(x, z)=\cos \left(\frac{(2 n+1) \pi x}{x_{0}}\right) e^{i z \sqrt{\frac{2 m E}{\hbar^{2}}-\left(\frac{(2 n+1) \pi}{x_{0}}\right)^{2}}}
\end{gathered}
$$

Equations (41) and (42) give a description of the propagation modes. As expected, and, assuming large $z$ propagation, they bear the form $\cos \left(\chi_{n} x\right) e^{i q_{n} z}$ for even modes and $\sin \left(\chi_{n} x\right) e^{i q_{n} z}$ for odd modes with the relationship: $\chi_{n}^{2}+q_{n}^{2}=\frac{2 m E}{\hbar^{2}}$. For a given incoming wave with $E>0$, the waveguide allows all propagation modes such that: $\chi_{n}^{2} \leq \frac{2 m E}{\hbar^{2}}$. Thus, for an incoming plane wave with a fixed energy $E=\frac{\hbar^{2}}{2 m} K_{i n}^{2}$ the highest order mode achievable is a function of the guide aperture and the incoming energy. The allowed energy levels in the guide do not depend on the angle of the incoming wavevector $\vec{K}_{i n}$. and this is a general fact.

By taking into account that both even and odd modes are allowed, the total number of modes in the waveguide will be approximately, twice the highest integer $n$ that generates a propagation mode:

$$
N_{\max } \approx \frac{X_{0}}{\pi} \sqrt{\frac{2 m E}{\hbar^{2}}}
$$






Figure 6. Number of allowed modes in waveguides having clad with infinite repulsive potential, depending on their aperture and incoming wave energy.

Figure 6 shows the results of the maximum number $\left(N_{\max }\right)$ of modes allowed in these waveguides as a function of the neutron incoming energy, $E$, and the aperture $\left(x_{0}\right)$ :

\section{Distribution of spatial frequencies}

According to Equation (39) an approximate analytical treatment is applicable for normal incidence, $K_{x}=0$ or small $K_{x}$. Moreover, the relative weight of each propagation mode arising for $K_{x} \neq 0$ will be analysed. We have performed some numerical computation enabling to calculate those weighting factors (the spectral distribution of the propagation modes) by adapting some quantummechanical approximation performed by Snyder and Love [25] to our problem. For brevity, we shall omit computational details. In Figure 7, we show the results as a function of the angle of the transverse wavevector: $\chi=\sqrt{K_{\text {in }}^{2}-q^{2}}=K_{\text {in }} \sin (\theta)$.

After Figure 7 results, the weighting factors have a maximum peak at an angle coinciding with the incidence angle of the incoming neutron wavevector. Indeed, most of the energy is carried along the waveguide with an angle similar to the one of the incoming wave. Moreover, there is some spread in the distribution of the incoming energy for other frequencies: the closest the value to the angle of the incident wave the higher the contribution. In an ideal case (i.e. no waveguide) the consistent result would be a delta function and thus, the incoming wave remaining unaltered. The spreading shape of the wavefunction is the

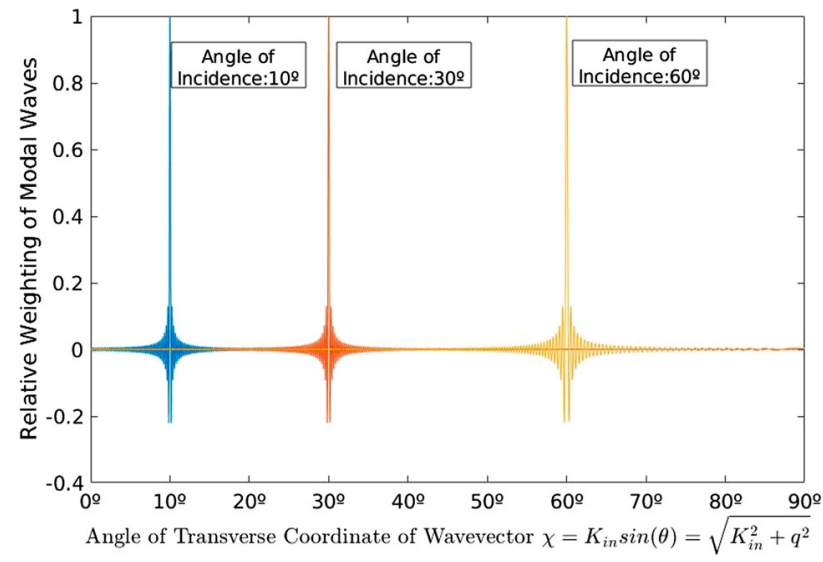

Figure 7. Relative weight factor distribution for three values of the angle of incidence. Energy of the incident wave: $E=0.025 \mathrm{eV}$.

expected physical behaviour, the net effect of the waveguide clad implying this spread in spatial frequencies. As a trivial result, the smaller the aperture the larger the spread in spatial frequencies for the incoming wave.

\section{Case for low energies}

Let us consider suitably small $E$, such that $x_{0} \sqrt{2 m E / \hbar^{2}} \ll$ 1 , so that both $x_{0} K_{x} \ll 1$ and $x_{0} K_{z} \ll 1$ hold. Then, it is expected that no propagation modes are excited along the semi-infinite waveguide. Focusing on $z>0$, with $z \gg x_{0}$ we apply Equations (16) and (17), in which we approximate the contributions due to $\mu_{1}(x)$ and $\mu_{2}(x)$ as in the derivation of Equation (25). In this case, we approximate 
all exponentials containing $x_{0}$ by unity:

$$
\frac{2 m}{\hbar^{2}} \frac{1}{2}\left(\mu_{3}(z)+\mu_{4}(z)\right) \simeq-2 \varphi_{\text {in }}(0, z)
$$

The latter, together with Equations (25) and (18) with $\varphi_{\text {in }}(x, z) \simeq \varphi_{\text {in }}(0, z)$ imply $\varphi(x, z) \simeq 0$ for long $z>$ 0 and any $-x_{0} / 2<x<x_{0} / 2$. This is fully consistent with Equation (39), with vanishing $\mu_{+}$and $\mu_{-}$. That is, the total wave function is extinguished and no propagation is excited, consistently. Strictly speaking, the above argument is only approximate, as long as we use only approximate expressions for $\mu_{i} i=1 \ldots 4$. However, for the values of $E$ assumed in this subsection, the extinction of the total wave function for large positive $z$ should hold as an exact statement, as no propagation modes are excited.

\section{Critical angle}

In geometrical optics, when describing the waveguiding effect, the acceptance or critical angle, $\theta_{c r}$ [26] is introduced. Then, as the waveguiding effect is described by means of total internal reflection, the guide will accept all incoming waves that obey $\theta_{\text {in }}<\theta_{c r}$. In our case, since we are supposing an ideal waveguide with infinite repulsive potential in the clad (i.e.: Dirichlet boundary conditions) one expects that the critical angle will be almost $\pi / 2$, that is, the waveguide allows almost any incident angle for incoming neutrons. In fact, the incident angle can be expressed as:

$$
\sin \phi=\frac{\mathrm{K}_{\mathrm{x}}}{\sqrt{K_{x}^{2}+K_{z}^{2}}}=\frac{K_{x}}{\sqrt{\frac{2 m E}{\hbar^{2}}}}
$$

In Equation (45) any possible $K_{x}$ gives rise to a possible $\chi_{n}$ value (both even and odd modes), with $\chi_{n}^{2} \leq$ $\frac{2 m E}{\hbar^{2}}, n=1 \ldots \frac{N_{\max }}{2}$, approximately. It would mean that the largest allowed $K_{x}$ would correspond to $n=\frac{N_{\max }}{2}$ and, so, $\sin \phi_{\max }=\frac{\max \left(K_{x}\right)}{\sqrt{\frac{2 m E}{\hbar^{2}}}}=\frac{\frac{\left(2 \pi \mathrm{N}_{\max } / 2\right)}{x_{0}}}{\sqrt{\frac{2 m E}{\hbar^{2}}}} \frac{\pi \mathrm{N}_{\max }}{x_{0} \sqrt{\frac{2 m E}{\hbar^{2}}}}$. By substituting according to Equation (43) we obtain: $\sin \phi_{\max }=$ $1 \Rightarrow \phi_{\max } \sim \pi / 2$. This is consistent with the above expectation.

\section{Discussion}

In the previous subsections, focusing on Physical System Description and Dirichlet Boundary Conditions, we have made a rigorous development of the equations to study the propagation of confined neutrons in semi-infinite waveguides with infinitely repulsive potential in the clad. The formulation through Equations (7) and (14)-(17) shows mathematical consistency, as one can check in the
Appendix. In it, we show that this approach yields the conservation of the probability current, as it should be expected.

Having developed such an analytical framework in Methods section, we have proceeded an analytical study onwards to make certain anzatz leading to approximate solutions of the problem. Let us suppose that the incoming neutron energy $E$ is such that only one propagation mode is excited. Then, an interesting issue is how the incident wave, as it propagates deeply along the interior of the semi-infinite waveguide and becomes extinguished with suitable contributions from $\Lambda_{1}+\Lambda_{2}+$ $\Lambda_{3}+\Lambda_{4}$ in Equation (18), generates the propagation mode. In other words, the problem is to compute the probability amplitude of the propagation mode generated in terms of the incoming neutron wave. Although a detailed study of this problem lies outside our scope here, the results of numerical computations regarding it have been given in the study of distribution of spatial frequencies.

The analysis of the mathematical system described here provides information useful enough to estimate the guiding efficiency only due to its geometry as acting as an upper limit. In any case, this formulation could be extended to other physical situation that could be described by means of a scalar wavefunction and in which Dirichlet conditions would play a key role.

As a general issue, the most ambitious simulation of neutron propagation would have to take into account different nuclear reactions with the media, incoherent scattering, thermal and epithermal absorption, etc. that will struggle with the basis of neutron optics approximation itself. In our case, our interest has been the analysis of confined propagation modes and their characterization. So far, neutron propagation has been described mostly by means of classical optics approximations but this propagation has not been extended, to the best of our knowledge, to the formalism of Green's functions to characterize these waveguides (and thus, propagation modes were not described but they were experimentally observed in [7-15]).

The case of finitely repulsive potential needs to be treated under a different formulation. This is a new project under development. Dirichlet boundary conditions are a zero-th order approximation for guides where the neutron suffers total reflection (i.e. for all waves with angle of incidence smaller than the critical angle). In these cases, our formulation will enable to study the neutron propagation along the waveguide at scales large compared to its wavelength and to avoid the computational challenge that penetration into the finitely repulsive clad represents $[27,28]$. Consistently, our next step would be a non-trivial extension of our present approach 
to more realistic conditions, where refractive indexes (or finite potentials) play a role, instead of Dirichlet boundary conditions. Another open problem is the development of new numerical algorithms noticeably improving the algorithms existing nowadays. A simulation of interest (the combination of a wavelength of thermal neutrons, $\lambda=1.8 \AA$ with apertures of the order of 100 $\mu \mathrm{m}$ ) would require high memory consumption and high computation times.

The probability for the penetration along a distance $x$ of a microscopic particle of mass $M$ and energy $E$ into a finitely repulsive clad with potential $V(>E)$ is proportional to $\exp \left(-2 \sqrt{\frac{2 M(V-E)}{\hbar^{2}} x}\right)$. Hence, that probability decreases as $M$ increases. The case of neutrons considered here is not a favourable one due to its relatively low mass, $m$, so that Dirichlet boundary conditions are just a zero-th order approximation. For atoms with $M \gg$ $m$, that probability decreases accordingly. In the study of the interactions of atoms with solid surfaces Dirichlet boundary conditions appear to be a rather adequate approximation. See for instance [29].

The degree of confinement of the neutron beam increases as the neutron energy $E$ decreases. An extreme situation of the latter kind, which would be of interest in connection with our present approach, could be the propagation of ultracold neutrons in waveguides. These neutrons, at temperatures of milikelvin, have an energy of the order of $10^{-7}-10^{-8} \mathrm{eV}$, that is, of the order of magnitude of the potential associated to a possible finitely repulsive media making up the clad. These neutrons suffer total internal reflection for any angle of incidence so that its wave function has a small (exponentially decaying) penetration in the clad. In this situation, the use of Dirichlet boundary conditions is more justified as a zeroth order description of the wave propagation inside the waveguide.

\section{Disclosure statement}

No potential conflict of interest was reported by the author(s).

\section{Funding}

We acknowledge Complutense University of Madrid (FECIEU-17-06), and Ministry of Science and Innovation (Project PGC2018-094684-B-C21) for financial support.

\section{Data availability statement}

The data that support the findings of this study (i.e.: data of performed simulations) are available from the corresponding author, [IMP] upon reasonable request.

\section{ORCID}

I. Molina de la Peña (D) http://orcid.org/0000-0002-5765-6858

M. L. Calvo (D) http://orcid.org/0000-0002-1303-319X

R. F. Alvarez-Estrada (D) http://orcid.org/0000-0003-0884-1157

\section{References}

[1] Byrne J. Neutrons. Nuclei and matter: an exploration of the physics of slow neutrons. Bristol: IoP Publishing; 1995.

[2] De Wames RE, Sinha SK. Possibility of guided-neutronwave propagation in thin films. Phys. Rev. B. 1973;7: 917-921.

[3] Sears VF. Neutron optics. Oxford: Oxford University Press; 1989.

[4] Alvarez-Estrada RF, Calvo ML. Neutron waveguides and applications. In: Calvo ML, Lakshminarayanan V, editor. Optical waveguides: from theory to applied technologies. Boca Raton, FL: CRC Press; 2007. p. 331-385.

[5] Alvarez-Estrada RF, Calvo ML. Neutron optics: fundamentals. In: Calvo ML, Alvarez-Estrada RF, editors. Advances in neutron optics: fundamentals and applications in materials science and biomedicine. Boca Raton (FL): CRC Press; 2019. p. 3-78.

[6] Alvarez-Estrada RF, Calvo ML. Neutron fibres: a possible application of neutron optics. J. Phys. D: Appl. Phys. 1984;17:475-502.

[7] Calvo ML, ALvarez-Estrada RF. Neutron fibres (II): some improving alternatatives and analysis of bending losses. J Phys D: Appl Phys. 1986;19:957-973.

[8] Feng YP, Marjkrzak CF, Sinha SK, et al. Direct observation of neutron-guided waves in a thin-film waveguide. Phys. Rev. B. 1994;49(15):10814-10817.

[9] Pogossian SP, Menelle A, Le Gall H, et al. Experimental observation of guided polarized neutrons in magneticthin-film waveguides. Phys. Rev. B. 1996;53:14359-14363.

[10] Menelle A, Pogossian SP, Le Gall H, et al. Observation of magnetic films neutron waveguides. Physica B. 1997;234236:510-512.

[11] Pogossian SP, Menelle A, Le Gall H, et al. Observation of neutron guided waves from the open end of a thin film waveguide interferometry. J. Appl. Phys. 1997;83:1159-1162.

[12] Pogossian SP, Le Gall H. Neutrons and and X-ray guided wave optics in nanostructures and multilayers. Recent Res. Devel. Optics. 2002;2:597-618.

[13] Kozhenikov SV, Khaydukov YN, Keller T, et al. Polarized neutron channeling as a tool for the investigations of weakly magnetic thin films. 2015; arXiv: 1511.07286v2/Cond-Mat.mtr1-Sci 24 Nov.

[14] Pleshanov N. Neutron spin optics: concepts, verification and prospects. In: Calvo ML, Alvarez-Estrada RF, editors. Advances in neutron optics: fundamentals and applications in material science and biomedicine. Boca Raton (FL): CRC Press; 2019. p. 205-237.

[15] Kumakhov MA, Sharov VA. A neutron lens. Nature. 1992;357:390-391.

[16] Chen H, Downing G, Mildner DFR, et al. Guiding and focusing neutron beams using capillary optics. Nature. 1992;357:391-393.

[17] Balian R, Bloch C. Distribution of eigenfrequencies for the wave equation in a finite domain: I. three-dimensional 
problem with smooth boundary surface. Ann. Phys. (N.Y.). 1970;60(2):401-447.

[18] Nieto-Vesperinas M. Propagación de luz y otras ondas electromagnéticas: Difracción y esparcimiento [light and electromagnetic waves propagation: scattering and diffraction]. In: Calvo ML, editor. Óptica avanzada [Advanced optics]. Barcelona: Ariel Ciencias; 2002. p. 41-82. Spanish.

[19] Nieto-Vesperinas N. Scattering and diffraction in physical optics. New York (NY): Wiley-Interscience; 1991.

[20] Mandel L, Wolf E. Optical coherence and quantum optics. Cambridge: Cambridge University Press; 1995.

[21] Bochner S, Chandrasekharan K. Fourier transforms. Princeton (NJ): Princeton University Press; 1949.

[22] Bedrosian E. A product theorem for Hilbert transforms. Santa Monia (CA): The Rand Corporation Memorandum; 1962. (RM-3439-PR)

[23] Pandey JN. The Hilbert transform of Schwartz distributions and applications. New York (NY): WileyInterscience; 1996. p. 89-113.

[24] Bracewell R. The Fourier transform and its applications. New York (NY): McGraw-Hill; 2000.

[25] Snyder AW, Love J. Optical waveguide theory. New York (NY): Springer; 1983.

[26] Molina de la Peña I, Calvo ML, Alvarez-Estrada RF. Neutron confinement and waveguiding. In: Calvo ML, Alvarez-Estrada RF, editor. Advances in neutron optics: Fundamentals and applications in materials science and biomedicine. Boca Raton, FL: CRC Press; 2019. p. 123-162.

[27] Molina de la Peña I, Calvo ML, Álvarez-Estrada RF. Focalizing slow neutron beams at and below micron scales: discussion on BNCT. Phosphorus, Sulfur. Silicon Relat Elems. 2019;194(10):956-966.

[28] Alvarez-Estrada RF, de la Peña I M, Calvo ML. Focalizing slow neutron beams at and below micron scales: discussion on BNCT (II). Phosphorus. Sulfur Silicon Relat Elems. 2018;193(2):64-73.

[29] García N, Cabrera N. New method for solving the scattering of waves from a periodic hard surface: solutions and numerical comparisons with the various formalisms. Phys. Rev. B. 1978;18:576-589.

[30] Newton RG. Scattering theory of waves and particles. 2nd ed New York (NY): Dover Books on Physics; 2013.

\section{Appendix: Conservation of Probability Current and Consequences}

The quantum-mechanical probability current along $z$ is, for $z>$ 0 and $z<0$ :

$$
J_{z}=J_{z}(z, x)=\frac{i \hbar}{2 m}\left[\varphi(z, x) \frac{\partial \varphi^{*}(z, x)}{\partial z}-\varphi^{*}(z, x) \frac{\partial \varphi(z, x)}{\partial z}\right]
$$

$\varphi^{*}(z, x)$ denotes the complex conjugate of $\varphi(z, x)$.

Conservation of the probability current along $z$, for any $x$ reads $\frac{\partial}{\partial z} J_{z}(z, x)=0$. It follows that the total probability flux across the whole $x$-axis, namely, $\int_{-\infty}^{\infty} d x J_{z}(z, x)$ is constant (independent on $z)$. Then, as $\varphi(z, x)$ vanishes in the clad:

$$
\left.\int_{-\infty}^{\infty} d x J_{z}(x, z)\right|_{z=+\infty}=\left.\int_{-x_{0} / 2}^{x_{0} / 2} d x J_{z}(x, z)\right|_{z=+\infty}
$$

Notice that $\left.\int_{-x_{0} / 2}^{x_{0} / 2} d x J_{z}(x, z)\right|_{z=+\infty}$ equals to the contribution of the propagation modes, provided that they be excited, otherwise that integral vanishes. We now turn to: $\left.\int_{-\infty}^{\infty} d x J_{z}(x, z)\right|_{z=-\infty}$. In order to evaluate the latter, we shall consider Equation (18) for $z<0$. In turn, $\Lambda_{1}+\Lambda_{2}$ and $\Lambda_{3}+$ $\Lambda_{4}$ are given by Equations (19) to (22), for $z<0$. The contribution of $\Lambda_{3}+\Lambda_{4}$ for $z \rightarrow-\infty$ is physically expected to vanish. Moreover, it can be easily invoked to vanish, by virtue of the Riemann- Lebesgue lemma. On the other hand, $\Lambda_{1}+\Lambda_{2}$ for $z \rightarrow-\infty$ is given by:

$$
\Lambda_{1}+\Lambda_{2}=\int \frac{d K_{x}^{\prime}}{2 \pi} e^{i K_{x}^{\prime} x} e^{-i z \sqrt{\frac{2 m E}{\hbar^{2}}-K_{x}^{\prime 2}}} g_{1}\left(K_{x}^{\prime}\right)
$$

where $g_{1}\left(K_{x}^{\prime}\right)=\frac{2 m}{\hbar^{2}} \frac{1}{2}\left[\int_{x_{0} / 2}^{\infty} d x^{\prime} \mu_{1}\left(x^{\prime}\right) e^{-i K_{x}^{\prime} x}+\int_{-\infty}^{-x_{0} / 2} d x^{\prime} \mu_{2}\left(x^{\prime}\right)\right.$ $\left.e^{-i K_{x}^{\prime} x}\right]$. The integration over $K_{x}^{\prime}$ yielding $\Lambda_{1}+\Lambda_{2}$ in Equation (A3) is now carried out only over the finite interval in which $0<\frac{2 m E}{\hbar^{2}}-K_{x}^{\prime 2}$. A direct computation shows easily that:

$$
\begin{aligned}
& \left.\int_{-\infty}^{\infty} d x J_{z}(x, z)\right|_{z=-\infty} \\
& \quad=\frac{K_{z} \hbar}{m} \int_{-\infty}^{\infty} d x-\frac{\hbar}{m} \int \frac{d K_{x}^{\prime}}{2 \pi} \sqrt{\frac{2 m E}{\hbar^{2}}-K_{x}^{\prime 2}} g_{1}^{*}\left(K_{x}^{\prime}\right) g_{1}\left(K_{x}^{\prime}\right)
\end{aligned}
$$

with $\sqrt{\frac{2 m E}{\hbar^{2}}+K_{x}^{\prime 2}}>0$

Again, the integration over $K_{x}^{\prime}$ being also carried out only over the finite interval in which $0<\frac{2 m E}{\hbar^{2}}-K_{x}^{\prime 2}$. Notice that $\int_{-\infty}^{\infty} d x$ in Equation (A4) is a divergent integral (which accounts for the infinite probability flux of the incoming plane wave $\varphi_{\text {in }}(z, x)$ across the whole $x$-axis). However, it is exactly cancelled by a completely similar divergent contribution (due to the infinite probability flux of the reflected plane wave), contained in $\frac{\hbar}{m} \int \frac{d K_{x}^{\prime}}{2 \pi} \sqrt{\frac{2 m E}{\hbar^{2}}-K_{x}^{\prime 2}} g_{1}^{*}\left(K_{x}^{\prime}\right) g_{1}\left(K_{x}^{\prime}\right)$. In fact, from Equations (19) and (20), approximating $\frac{2 m}{\hbar^{2}} \frac{1}{2} \mu_{1}(x) \sim-\varphi_{\text {in }}$ $(x, 0)$ for $x \gg x_{0} / 2, \frac{2 m}{\hbar^{2}} \frac{1}{2} \mu_{2}(x) \sim-\varphi_{\text {in }}(x, 0)$ for $x \ll-x_{0} / 2$ (ie: total reflection) and the sum of $\int_{-\infty}^{-x_{0} / 2} d x^{\prime}$ and $\int_{x_{0} / 2}^{\infty} d x^{\prime}$ by $\int_{-\infty}^{\infty} d x^{\prime}$ like in Equation (27) one has:

$$
\begin{aligned}
\Lambda_{1}+\Lambda_{2} & \approx-\int d K_{x}^{\prime} \delta\left(K_{x}-K_{x}^{\prime}\right) e^{i K_{x}^{\prime} x} e^{-i z \sqrt{\frac{2 m E}{\hbar^{2}}-K_{x}^{\prime 2}}} \\
& =-e^{i K_{x} x} e^{-i z K_{z}}
\end{aligned}
$$




$$
\begin{aligned}
\Rightarrow g_{1}\left(K_{x}^{\prime}\right) & \simeq \int_{-\infty}^{\infty} d x^{\prime} e^{i x^{\prime}\left(K_{x}-K_{x}^{\prime}\right)}=-2 \pi \delta\left(K_{x}-K_{x}^{\prime}\right) \\
& \Rightarrow \int \frac{d K_{x}^{\prime}}{2 \pi} \sqrt{\frac{2 m E}{\hbar^{2}}-K_{x}^{\prime 2}} g_{1}^{*}\left(K_{x}^{\prime}\right) g_{1}\left(K_{x}^{\prime}\right) \\
& \approx \sqrt{\frac{2 m E}{\hbar^{2}}-K_{x}^{2}} \int_{-\infty}^{\infty} d x
\end{aligned}
$$

which leads to the cancellation of the divergent term: $\frac{K_{z} \hbar}{m} \int_{-\infty}^{\infty} d x$ in Equation (A.4).
The net outcome is that both sides of $\frac{K_{z} \hbar}{m} \int_{-\infty}^{\infty} d x-$ $\frac{\hbar}{m} \int \frac{d K_{x}^{\prime}}{2 \pi} \sqrt{\frac{2 m E}{\hbar^{2}}-K_{x}^{\prime 2}} g_{1}^{*}\left(K_{x}^{\prime}\right) g_{1}\left(K_{x}^{\prime}\right)=\left.\int_{-x_{0} / 2}^{x_{0} / 2} d x J_{z}(x, z)\right|_{z=+\infty} ^{-\infty}$ are finite. The left-hand side of this equation describes finite corrections to total reflection. The resulting equation, after the cancellation of divergences, turns out to represent the actual counterpart for semi-infinite waveguides with Dirichlet boundary conditions, of the standard optical theorem in scattering theory (see Newton [30]). 